\title{
DOSSIÉ: LA TRADUCCIÓN EN LA ENSEÑANZA Y EN EL APRENDIZAJE DE LENGUAS EXTRANJERAS ${ }^{I}$
}

\author{
Maria Cristina Reckziegel Guedes EVANGELISTA ${ }^{2}$ \\ Paula Tavares PINTO ${ }^{3}$ \\ Odair Luiz NADIN ${ }^{4}$
}

\section{PRESENTACIÓN}

El objetivo de la Revista EntreLínguas es "Publicar artículos, relatos de experiencias, reseñas críticas y entrevistas de investigadores brasileños y extranjeros sobre la enseñanza y el aprendizje de lenguas extranjeras modernas (LEM), para promover el debate sobre los procesos teórico-metodológicos de la enseñanza de LEM."

En neste contexto, presentamos el volumen temático de la Revista EntreLínguas (V. 3, n. 2), sobre el tema "La traducción en la enseñanza y en el aprendizaje de lenguas extranjeras". Ese tema aún es motivo de controversias y sigue siendo un tabu para muchos profesores de lenguas extranjeras e investigadores, aunque los estudios sobre esa temática nunca haya sido interrumpidos, como muestran, por ejemplos, la copilación sobre el uso de la traducción y la enseñanza de lenguas extranjeras en la Unión Europea, preparado por Pyn; Malmkjær y Gutiérrez-Colón Plana (2013).

Entre los trabajos ya publicados, se observa una tendencia a presentar en primer lugar una retrospectiva sobre los métodos y abordajes de enseñanza de lenguas extranjeras, seguida de la justificativa para el uso de la traducción, como muestram los siguientes trabajos, presentados en orden cronológico crecente: Terra (2010); Tecchio; Bittencourt (2011); Briks (2012), Liberatti (2012); Souza Corrêa (2014), entre otros. Los textos de este volumen se vuelven principalmente para la discusión seguidamente (a nível) posterior a esa fase de justificativas, presentando razones concretas para la

${ }^{1}$ Traducción en español: Profesor Doctor Odair Luiz Nadin y Profesora Doctora Nildicéia Aparecida Rocha.

${ }^{2}$ Universidad Estatal Paulista (Unesp), Facultad de Ciencias y Letras, Araraquraa - SP - Brasil. Profesora Doctora del Departamento de Letras Modernas. Correo electrónico: macrisevangelista @fclar.unesp.br.

3 Universidad Estatal Paulista (Unesp), Instituto de Biociencias, Letras y Ciencias Exatas, São José do Rio Preto - SP - Brasil. Profesora Doctora del Departamento de Letras Modernas. Correo electrónico: paula@ibilce.unesp.br.

${ }^{4}$ Universidad Estatal Paulista (Unesp), Facultad de Ciencias y Letras, Araraquara - SP - Brasil. Profesor Doctor del Departamento de Letras Modernas. Correo electrónico: odairnadin@ fclar.unesp.br 
utilización de la traducción y algunas formas de insertarla en el contexto de enseñanza y aprendizaje.

Valdecy Oliveira Pontes y Livya Lea Oliveira Pereira, en el artículo La traducción funcional y la variación linguística: el uso de secuencia didáctica en la enseñanza de lenguas, relatan sobre la elaboración de una secuencia didáctica en la que se traducen fragmentos de piezas treatrales de diferentes países hispánicos, teniendo por enfoque las formas de tratamento del español y del portugués.

Elisa Figueira de Souza Corrêa presenta el artículo La traducción Pedagógica: experimentos y ejercicios para su uso en clase y postula que la habilidad traductoria puede ser puesta junto a las cuatro destrezas tradicionales, leer, escribir, hablar y comprender y, así, contribuir al desarrollo de la consciencia lingüística del aprendiz.

Sinara de Oliveria Branco y Luciana Soares dos Santos elaboraron el artículo El uso de actividades de traducción intersemiotica e interlingual en una clase de lengua inglesa como $L E$, en que buscan evidenciar la eficiencia del uso de la traducción como herramienta de enseñanza de lengua inglesa, tomando por base las categorías de traducción propuestas por Jakobson (1958/2000) y siguiendo una metodologia de naturaleza cualitativa y de investigación-acción.

Aline Cantarotti presenta Data-driven learning, traducción y Secretariado Ejecutivo: propuesta de abordaje de autoestudio para aprendices de inglés. $\mathrm{Su}$ investigación acerca la metodología del trabajo de linguística de corpus al ámbito del aprendizaje de lengua extranjera, llevando el aprendiz a descobrir por si mismo las características léxico-gramaticales de la lengua que estudia.

Viviane Cristina Poletto Lugli, autora del artículo La expresión de verbos modales en la producción del género decisión: contribuciones para la enseñanza de lengua extranjera, propone la comparación entre fragmentos de textos de ese género en portugués, español e inglés, uniendo los estudios de la lengua extranjera y de los géneros textuales.

Paolo E. Balboni, en Traducción en el aprendizaje de lenguas: un abordaje 'para qué', busca evaluar cómo la traducción puede traer benefícios a las diferentes habilidades relacionadas a las competencias lingüísticas, extralingüísticas, sociopragmáticas e intercultural de los aprendices de lengua extranjera.

Bruna Di Sabato y Bronwen Hughes, en el artículo Traducción y enseñanza de lengua extrajera, un punto de vista teórico y práctico: el escenario italiano, discurren sobre el papel de la traducción en currículos de universidades italianas y muestran 
algunas actividades de traducción que pueden ser desarrolladas por los aprendices de lenguas.

Joselma Maria Noal; Artur Emílio Alarcon Vaz y Daniele Corbetta Pilleti proponen discusiones sobre La traducción literaria en la enseñanza de lenguas. Su investigacón parte de la traducción de cuentos de la escritora argentina Juana Manuela Gorriti por los aprendices y analiza dificultades en la traducción, relacionadas a los ámbitos gramatical, lexical y estilístico, mostrando que ese trabajo también acerca el aprendiz a cuestiones culturales.

Krisztina Zimanyi presenta el artículo Pasaporte a la traducción en la clase de una lengua extranjera: una propuesta para la traducción audiovisiual de Coralini en clases de inglês como LE/ESOL. Las actividades propuestas por la autora incluyen la traducción intralingual, interlingual e intersemiótica (JAKOBSON, 1959/2000) y buscan motivar a los aprendices, promoviendo su autonomía.

Glauber Lima Moreira presenta la entrevista realizada a la profesora Dra. Janet DeCesaris de la Universitat Pompeu Fabra (UPF), intitulada El papel de la traducción en la enseñanza de lenguas extranjeras modernas. La entrevistada entiende la traducción principalmente como una herramienta didáctica, que permite la comparación entre las lenguas, promoviendo la conscientización sobre cuestiones linguísticas.

\section{REFERÊNCIAS}

BRIKS, F. J. P. Tradução: Ferramenta eficaz no ensino-aprendizagem de línguas estrangeiras para o aluno do ensino superior. In: Belas Infiéis, v. 1, n. 1, p. 153-167, 2012.

JAKOBSON, R. On linguistic aspects of translation. In: VENUTI, L. The Translation Studies Reader. London/New York: Routledge, 113-118, 1959/2000.

LIBERATTI, E. A tradução na sala de aula de LE: (des)construindo conceitos. In: Entrepalavras, Fortaleza, ano 2, v. 2, n. 1, p. 175-187, 2012.

PYM, A.; GUTIÉRREZ-COLÓN PLANA, M. d. M.; MALMKJAER, K. Translation and language learning: the role of translation in the teaching of languages in the European Union. A Study. Directorate-General for Translation - European Commission. 15 July 2013. 
RIDD, M. Bibliografia de referência sobre tradução no ensino e aprendizagem de línguas. In: Horizontes de Linguística Aplicada, v. 8, n. 2, p. 255-296, 2009.

SOUZA CORRÊA, E. F. de. A língua materna e a tradução no ensinoaprendizagem de língua não-materna: uma historiografia crítica. Tese de doutorado. PUC, Rio de Janeiro, 2014.

TECCHIO, I.; BITTENCOURT, M. J. G. A tradução no ensino-aprendizagem de línguas estrangeiras. In: Revista Magistro, v. 2, n. 1, p. 152-165, 2011. Disponível em:

TERRA, M. R. Tradução \& aprendizado de língua estrangeira: o ponto de vista do aluno. Trab. Ling. Aplic., Campinas, v. 49, n.1, p. 69-85, jan./jun. 2010.

\section{Cómo citar este texto:}

EVANGELISTA, Maria Cristina Reckziegel Guedes.; PINTO, Paula Tavares.; NADIN, Odair Luiz. Presentación Dossié: La traducción en la enseñanza y en el aprendizaje de lenguas extranjeras. Rev. EntreLínguas, Araraquara, v.3, n.2, p. 149-152, jul./dez. 2017. Disponível em: <https://doi.org/10.29051/rel.v3.n2.2017.10798>. E-ISSN: 24473529. 